\title{
A FOURIER-BASED METHOD FOR DETECTING CURVED MICROTUBULE CENTERS: APPLICATION TO STRAIGHTENING OF CRYO-ELECTRON MICROSCOPE IMAGES
}

\author{
S. Blestel ${ }^{1}$, C. Kervrann ${ }^{1,2}$, D. Chrétien ${ }^{3}$ \\ (1) INRIA, Centre de Rennes Bretagne Atlantique, IRISA/Univ. Rennes I, F-35042 Rennes \\ (2) INRA, UR341 Mathématiques et informatique appliquées, F-78352 Jouy-en-Josas \\ (3) CNRS, UMR 6026, IFR 140, F-35042 Rennes
}

\begin{abstract}
In this paper, we propose two sensitive contributions to automatically determine the local orientations and centers of short segments of microtubules in cryo-electron microscope images. Indeed, to our knowledge, the methods to determine the local centers of helices are not relevant for non centro-symmetric helices (e.g. 13-protofilament microtubules). The proposed algorithm exploits the helical symmetry of microtubules and the corresponding properties in the Fourier domain, so it can be easily extended to process other helical objects. Experimental results demonstrate that center locations are estimated with an accuracy of lower than one pixel. We applied the algorithm to automatically straighten images of curved microtubules with odd numbers of protofilaments, and to improve 3D reconstructions of microtubules using back-projection methods.
\end{abstract}

Index Terms - Fourier transforms, cryo-electron microscopy, image orientation analysis, Bessel functions, microtubules

\section{INTRODUCTION}

Microtubules are long tubes of $\sim 25$ nanometers in diameter present in all eukaryotic cells. They play fundamental roles in cells life, in particular during their division since they are involved in chromosome segregation. The understanding of their structure and assembly mechanisms is of major importance not only in fundamental biological research, but also in medicine since microtubules are a major target of anti-mitotic drugs used in cancer therapy. The study of their molecular architecture is possible by cryo-electron microscopy, which enables observation of biological specimens frozen at liquid nitrogen temperature in their native state. The images obtained are projection views of the specimens perpendicular to the electron beam that have a resolution on the order of a few (tenth of) Angströms ( $\AA$ ).

In this study, we are interested in longitudinal projections of microtubules (i.e. perpendicular to the microtubule axis). Microtubules are composed of identical subunits that arrange together to form a helical lattice (see Figure 2). Because of their symmetry, most of their information can be retrieved from their Fourier spectrum. However, due to their flexibility, microtubules are generally curved and their Fourier transform can no longer be used for symmetry analysis.

Consequently, image processing algorithms have been developed to straighten helical polymers observed by electron microscopy. These algorithms straighten a spline curve fitted to a set of nodes located at the center of the object [1]. Manual selection of local centers on microtubule images is a hard task because the views have low contrasts and low signal-to-noise ratios. Alternatively, centers can be automatically computed on short segments by cross-correlation between the segment and its symmetric image with respect to the microtubule axis. But this method requires centro-symmetric microtubules, i.e. microtubules which projection views are symmetrical with respect to their longitudinal axis. To our knowledge, all packages and softwares developed for helical analysis and straightening are based on the pioneering study of Egelman [1]. In these packages, the nodes are first selected manually, and then refined using different approaches. In the Brandeis Helical package [2], the straightened microtubule and its Fourier transform are displayed while nodes are selected, which can then be spatially adjusted by looking at the quality of the diffraction pattern. In the Ruby-Helix package [3], the nodes are adjusted by cross-correlation between the microtubule projection and its symmetric with respect to its longitudinal axis, which may be not appropriate for non centrosymmetric microtubules. In the "Straighten Curved Objects" plug-in of Image J [4], the nodes are adjusted by cross-correlation between the global axial average of the straightened microtubule and the local average of the updated node neighborhood. Yet, this approach does not guarantee the precise determination of the microtubule local centers.

In this paper, we propose two contributions to improve the determination of local orientations and centers of a curved microtubule, centro-symmetric or not. Our approach relies on a criterion computed in the Fourier domain and based on the global cylindrical shape of the helix. This criterion is independent of the parity of the helix, it is thus relevant for microtubules with even and odd numbers of protofilaments. In section 2, we present the Fourier transform of helices and the details of our approach. The method is then evaluated on synthetic data, and applied to real data to straighten curved microtubules (Section 3). Our method is relatively easy to use and an ImageJ plug-in will be freely available for users.

\section{METHOD}

\subsection{Fourier transform of helices}

In the following, we consider Cartesian coordinates $(x, y, z)$ and cylindrical coordinates $(r, \phi, z)$ in the real image space, where $z$ denotes the helix axis, $r$ the radius and $\phi$ the azimuthal rotation (rotation around $z$ ). In the Fourier space we use the same coordinate systems with letters in upper cases: $(X, Y, Z)$ and $(R, \Phi, Z)$.

A helical wave is a plane wave curved onto a cylinder whose intensity varies between -1 and +1 . A helical wave can be defined by three parameters :

- the radius $\rho$ of the cylinder,

- the rotational frequency $n$ (or helix start number), that is the number of repeats of the wave while rotating azimuthally (i.e. in a plane perpendicular to $z$ ),

- the pitch $p$ corresponding to the axial distance traversed for the wave to rotate $360^{\circ}$ around the helix axis (see Figure 2). 


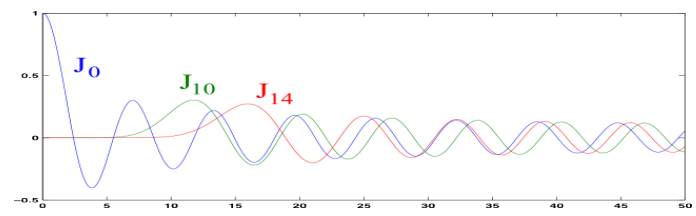

Fig. 1. Bessel functions for different orders.

The Fourier transform of a helical wave is defined as:

$$
\mathcal{F}(R, \Phi, Z= \pm n / p)=J_{n}(2 \pi R \rho) \exp ^{(\jmath n(\phi+\pi / 2)}
$$

and consists of a pair of discs, called layer-lines, perpendicular to the $Z$ axis and located axially at $Z= \pm n / p$; it is null elsewhere [5]. The magnitude of the spectrum is given by a Bessel function of order $n$, noted $J_{n}$ (see Figure 1). The magnitude is independent of the azimuth $\Phi$, and only the phase varies with $\Phi$.

If we consider the uniform density wave as a particular helical wave such as $n=0$ and $n / p=0$, the density distribution of any helical object can be conceived as a sum of helical waves [6]. Therefore its Fourier transform will be the sum of the transforms of each individual wave.

The projection theorem [7] states that the Fourier transform of a projected density corresponds to a section through the threedimensional Fourier transform of the density. This section passes through the origin and is perpendicular to the direction of the projection. Therefore the Fourier transform of the projection, perpendicular to the $z$ axis, of a helical object, corresponds to a section through the transform of the three-dimensional helix that contains the $Z$ axis. The two-dimensional Fourier spectrum consists of a sum of layer-lines located at $Z= \pm n / p$ corresponding to each helical wave of the helix.

\subsection{Estimation of microtubule center from Fourier spectrum}

We propose a method to determine the center of a straight microtubule whose orientation is known. This method relies on the analysis of the phases in the Fourier domain. We first apply a simple homomorphic filter [8] to the input image to remove non-uniform illumination effects, but other more sophisticated methods could be also investigated [9]. We then present the centering method and its initialization step which relies on phase-correlation. Finally, a method to get the microtubule orientation is proposed.

Correction of non-uniform illumination. Cryo-electron microscopy images have a spatially varying contrast due to the varying thickness of the ice. This non-uniform illumination is characterized by a slow variation of the mean intensity of the image which modifies the phases of the transform at low frequencies and must be corrected. The homomorphic filter [8] enables to extract illumination $I_{i}$ from an observed image of intensity $I$ defined as $I=I_{i} I_{0}$, where $I_{0}$ is the desired image. Taking the logarithm, the multiplicative relation is transformed into an additive one. The log-illumination $\log \left(I_{i}\right)$ is obtained by applying to $\log (I)$ a low-pass Gaussian filter with a high standard deviation of the order of the microtubule diameter. The log-illumination is subtracted from $\log (I)$ to get an estimation of $\log \left(I_{0}\right)$. Finally, our algorithm is applied to the corrected image $f=\exp \left(\log \left(I_{0}\right)\right)$.

Method for center determination. Microtubules are known to be composed of basic subunits, the $\alpha \beta$ tubulin protein, a dimer composed of $\alpha$ - and $\beta$-tubulin. Due to image resolution, the two
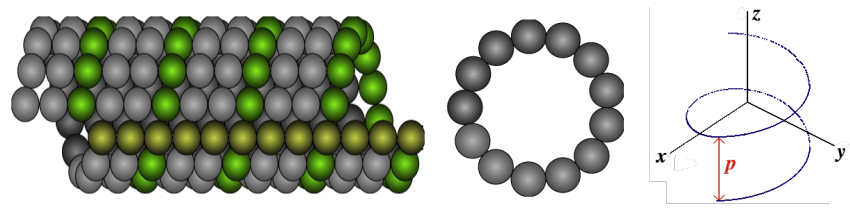

Fig. 2. Longitudinal (left) and top view (center) of the structure of a microtubule (in yellow: one of the 13 protofilament helices $\left(n_{\text {pro }}=13\right)$; in green: one of the 3 tubulin helices $\left.\left(n_{\text {tub }}=3\right)\right)$ and representation of a helical wave (right) where $p$ denotes the helix pitch. Note that only tubulin monomers have been depicted for clarity, since the tubulin molecule is indeed a dimer.

monomers are undistinguishable, so in the following tubulin will refer to the tubulin monomer. The tubulin proteins arrange to form protofilaments [10], that associate laterally in hollow cylinders to form microtubules. This produces a helical lattice whose basic helices correspond to the protofilaments and the tubulins (see Figure 2). Depending on the microtubule configuration, the "protofilament" basic helix has a very large or infinite pitch and a rotational frequency $n_{\text {pro }}$ generally ranging from 11 to 15 see [10]). The "tubulin" helix has a low pitch and a rotational frequency $n_{\text {tub }}$ whose value is generally ranging from 2 to 4 .

The Fourier transform of a straight microtubule consists of a set of layer-lines perpendicular to the $Z$ axis. The protofilament and tubulin helices induce two main Bessel functions $J_{n_{\text {pro }}}$ and $J_{n_{\text {tub }}}$ located respectively at $Z= \pm n_{\text {pro }} / p_{\text {pro }}$ (near or on the equator) and $Z= \pm n_{\text {tub }} / p_{\text {tub }}$, where $p_{\text {pro }}$ and $p_{\text {tub }}$ denote respectively the pitch of the "protofilament" and the pitch of the "tubulin" helices. Furthermore, the global cylindrical structure of the microtubule gives rise to a Bessel function of order zero located at the equator $(Z=0)$ of the transform. Additional secondary layer-lines are also present that correspond to secondary helices. Indeed there is an infinity of helices in a helical lattice (by taking any two points of the lattice and its parallels), but due to the microscope resolution only a few layer-lines appear in the Fourier spectrum.

To determine the center of a straight microtubule whose $Z$ axis is known, we consider the phases of the zero-order Bessel function in the Fourier spectrum. The $J_{0}$ Bessel function corresponds to the global cylindrical shape of the microtubule, so the criterion does not depend on the symmetry of the helix projection. If the microtubule is centered, the phase is $\psi=0$ when $J_{0}$ is positive and $\psi=\pi$ when $J_{0}$ is negative (see Figure 1). If the microtubule is not centered, the phases will deviate from 0 or $\pi$ because a translation in the real space corresponds to a phase shift in the Fourier space. Because we consider the Bessel function of order 0 , that corresponds to the cylindrical uniform wave, an axial translation does not modify the wave and its transform. Only the translations perpendicular to the $z$ axis modify its phases. Hence, if $g(x, z):=f(x+t, z)$, we have

$$
\begin{aligned}
\mathcal{G}(X, Z) & =\exp ^{2 \jmath \pi X t} \mathcal{F}(X, Z), \\
\psi_{\mathcal{G}}(X, Z, t) & =2 \pi X t+\psi_{\mathcal{F}}(X, Z),
\end{aligned}
$$

where $\mathcal{G}$ is the Fourier transform of $h$ and $\psi_{\mathcal{G}}(., .,$.$) denotes the$ phase of $\mathcal{G}$. For given $X$ and $Z$, the function $\psi_{\mathcal{G}}(X, Z, t)$, noted $\psi_{\mathcal{G}}(t)$, is piecewise linear. The optimal translation $\hat{t}$ for which the microtubule is centered (i.e. $\psi_{\mathcal{G}}(X, Z, \hat{t})=0$ or $\pi$, depending on the sign of $J_{0}$ ) can be determined experimentally by applying successive translations to the microtubule, perpendicular to its $z$ axis, while analyzing the phase. To get non integer coordinates, bilinear interpolation is used in the whole algorithmic procedure. 
The Bessel function of order 0 is on the equator of the Fourier spectrum. Nevertheless, additional Bessel functions in the local neighborhood of this layer-line may modify the phases $(0$ or $\pi$ ) of the $J_{0}$ function. Moreover, in real images, the phases of the Fourier spectrum can be corrupted by noise. Another limitation is that $\psi_{\mathcal{G}}(t)$ is $1 / X$ periodic $(1 / X \sim 0.6 \rho$, where $\rho$ is the radius of the microtubule), so the algorithm has to be initialized with a coarse translation $\hat{t}_{0}$ to guarantee that the relevant extremum is determined.

To avoid modification of the phases due to other Bessel functions located near the equator, only the first three oscillations of the magnitudes of the zero-order layer-line are used to compute the microtubule center. Indeed, Bessel functions can add to $J_{0}$ on the zeroorder layer-line if they are located near or on the equator. These Bessel functions are induced by the protofilament helix. They have a high order (11 to 15 ), so they only appear at high frequencies (see Figure 1). Thus one can consider that the three first oscillations of the magnitudes of the zero-order layer-line correspond only to $J_{0}$ and that the corresponding phases are not modified.

To increase the robustness to noise, we consider the phases of several spectral components whose magnitudes are high compared to less meaningful magnitudes. Indeed, noise is spatially unorganized so its Fourier transform has small magnitudes and does not modify notably the phases of the spectral components with high magnitudes.

Initialization of the algorithm. Phase correlation [11] allows to determine the center of straight centro-symmetric helices (e.g. microtubules with 12 or 14 protofilaments). For the non centro-symmetric helices, it provides a coarse estimation of their center. To initialize the algorithm, we compute the phase correlation between the microtubule image $f$ and its symmetric image with respect to its $z$-axis, noted $\bar{f}$. The optimal translation to superpose $f$ and $\bar{f}$ is then $2 \hat{t}_{0}$, where $\hat{t}_{0}$ is an estimate of the translation to center $f$. The phase correlation of $f$ and $\bar{f}$ is defined as the inverse Fourier transform of

$$
\frac{\mathcal{F}^{*}(\xi, \eta) \overline{\mathcal{F}}(\xi, \eta)}{|\mathcal{F}(\xi, \eta)||\overline{\mathcal{F}}(\xi, \eta)|}
$$

where $\mathcal{F}$ and $\overline{\mathcal{F}}$ are respectively the Fourier transforms of $f$ and $\bar{f}$, $\xi$ and $\eta$ denote the frequencies, $*$ denotes complex conjugate and $|$. is the magnitude of the transform. If $\bar{f}(x, z)=f\left(x-2 \hat{t}_{0}, z\right)$ then the phase correlation is an impulse at position $\left(2 \hat{t}_{0}, 0\right)$. Images of microtubules have a low signal-to-noise ratio, and the frequencies with low magnitudes are likely to be corrupted by noise so they are masked and do not contribute to the phase correlation. High frequencies are also masked because they correspond mainly to noise, due to the attenuation of the contrast transfer function.

Angular correlation for microtubule orientation. To compute the microtubule center, we have supposed that its orientation was known. This orientation can be automatically determined by examining the orientation of the layer-lines in the Fourier domain, known to be perpendicular to the microtubule axis. We thus compute the angular correlation between the magnitude images $|\mathcal{F}|$ and $\left|\mathcal{F}^{\prime}\right|$, where $\mathcal{F}$ is the Fourier spectrum of the microtubule image $f$ and $\left|\mathcal{F}^{\prime}\right|$ is the mirror image of $|\mathcal{F}|$. The angular correlation between $|\mathcal{F}|$ and $\left|\mathcal{F}^{\prime}\right|$ is defined as the set of correlation coefficients between $|\mathcal{F}|$ and $\left|\mathcal{F}_{\theta}^{\prime}\right|$ for $\theta$ ranging from 0 to $180^{\circ}$, where $\left|\mathcal{F}_{\theta}^{\prime}\right|$ is $\left|\mathcal{F}^{\prime}\right|$ rotated by $\theta$. The best correlation is obtained when the layer-lines of $\mathcal{F}$ and $\mathcal{F}_{\hat{\theta}}^{\prime}$ are superposed. The estimate $\hat{\theta}$ of the microtubule angular deviation is

$$
\underset{\theta}{\arg \min } \frac{1}{\sigma_{\mathcal{F}} \sigma_{\mathcal{F}_{\theta}^{\prime}}} \sum_{(\xi, \eta)}\left(|\mathcal{F}(\xi, \eta)|-\mu_{\mathcal{F}}\right)\left(\left|\mathcal{F}_{\theta}^{\prime}(\xi, \eta)\right|-\mu_{\mathcal{F}_{\theta}^{\prime}}\right),
$$

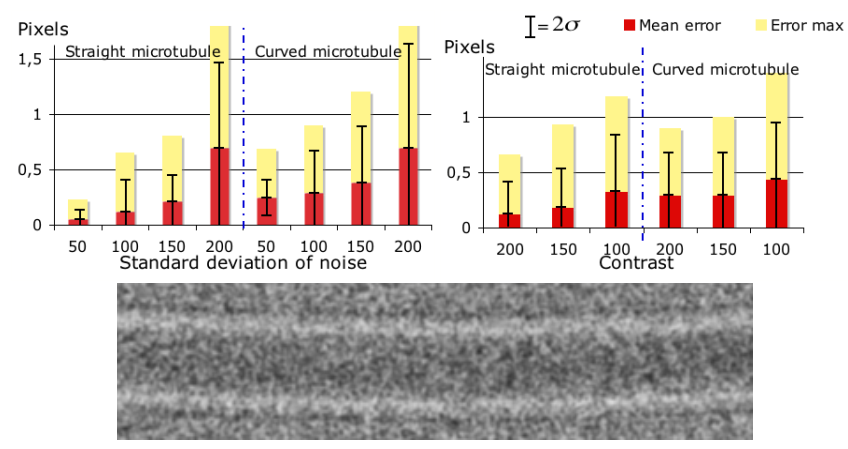

Fig. 3. Evaluation of the centering method (top) on synthetic data for different values of noise (and a contrast of 200) and contrast (and a noise of standard deviation 100). The synthetic image (bottom) has a contrast of 200, an additive noise of standard deviation 100, and was convolved with a gaussian of standard deviation 1 pixel.

where $\mu_{\mathcal{F}}$ and $\sigma_{\mathcal{F}}$ are the mean and standard deviation of the magnitudes $|\mathcal{F}|$ of $\mathcal{F}$.

For more accuracy, we perform the rotation of angle $\theta$ in the real image domain, which avoids rotationally dependent aliasing. Nevertheless, the angular correlation is performed in the spectral domain (see (4)). Moreover, as for computation of phase correlation, we select low and medium frequencies to compute angular correlation.

\section{EVALUATION AND APPLICATIONS}

\subsection{Evaluation on synthetic data}

To evaluate the accuracy of our method, we compared the estimated centers on artificial images with ground truth. Our method only considers the oscillations of the zero-order layer-line that correspond to the $J_{0}$ Bessel function. Accordingly, microtubules are represented with tubes and only the three first oscillations of the zero-order layerline are considered to estimate its center (see section 2.2). The synthetic data is the projection of a tube corrupted with an additive white Gaussian noise and convolved with a Gaussian filter of standard deviation 1 pixel. This convolution is needed to represent the correlation of noise observed in real cryo-electron microscope images. We evaluated the algorithm on a straight and a curved tube (with a curvature of approximately $3.510^{-4}$ pixels), for different image contrasts and standard deviations of the noise. The statistics presented in Figure 3 were computed from 20 estimated centers. The contrast is defined as the maximal intensity of the projected tube minus its minimal intensity. Typically, real images have a contrast of 200 and a Gaussian noise with a standard deviation of 100 convolved by a Gaussian kernel of standard deviation of 1 pixel (see Figure 3).

On synthetic data with noise and contrast close to real data, the average estimation error is lower than 0.3 pixel.

\subsection{Straightening images of curved microtubules}

The microtubules embedded in vitreous ice are well preserved, but still display varying amounts of curvature. To analyze these images, it is therefore necessary to straighten them. Straightening is generally done by fitting a curve to the local centers of the microtubule; interpolation-based straightening is then applied by determining a new grid normal to this curve [1]. We used our approach to automatically compute the microtubule centers in order to fit the spline 


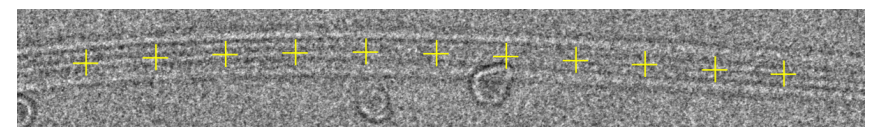

(a) Original microtubule with several estimated centers (yellow crosses)

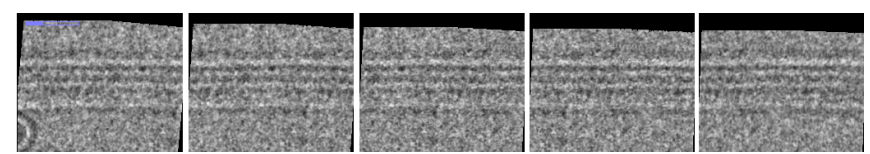

(b) Zoom in first segments oriented and centered with our method

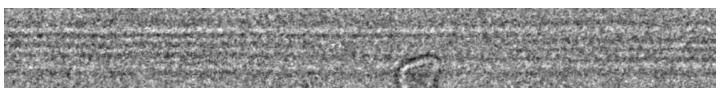

(c) Straightened microtubule

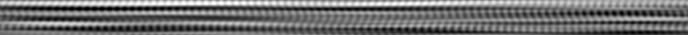

(d) Straightened microtubule (c) filtered in the Fourier domain (only Bessel functions $J_{0}, J_{13}, J_{3}$ and $J_{10}$ are selected)

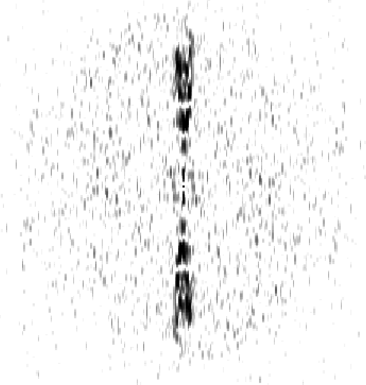

(e) Fourier transform of (a)

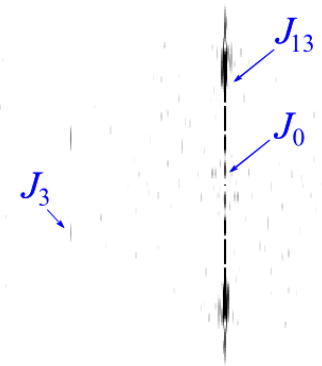

(f) Fourier transform of (c)
Fig. 4. Straightened 13-protofilament microtubule (high densities) from centers computed using our algorithm.

curve. As the curvature of the microtubule is small, we can consider that it is locally linear and apply our method.

The algorithm estimates the orientation and the center of short segments along a microtubule. The successive segments do not need to overlap. Nevertheless, the segment must be carefully chosen: segments have to be short enough to ensure that the microtubule inside is straight, and long enough to get well-defined layer-lines in the Fourier domain. The centering method is initialized with the phase correlation method (see section 2.2) on the first segment. For the next segments, the coarse center can be computed from the fine center determined on the last segment and its estimated orientation.

Several experts validated the algorithm on centro and non centro-symmetric microtubules. Figure 4 shows an example of this method applied on a non centro-symmetric microtubule image. Pixels have a size of $\sim 4 \AA$ at the specimen level. Segments are 125 pixels length $(\sim 50 \mathrm{~nm})$ and overlap on 50 pixels. The orientation was computed with angular correlation, with an angular discretization of 0.1 degree. We compute the centers with the phases corresponding to the higher magnitudes of the three first oscillations of the zero-order layer-line, with a translational step of 0.05 pixel.

Figure $4 \mathrm{c}$ shows the straightened 13-protofilament microtubule. The Fourier transform of the microtubule (Figure 4e) is composed of blurred layer-lines with several orientations. The transform of the straight microtubule (Figure 4f) has sharp and more intense layer-lines which can be well separated for further analysis such as filtering (Figure 4d) or three-dimensional reconstruction by back-projection (Figure 5). The 3D reconstructions can be used to determine microtubule polarity.
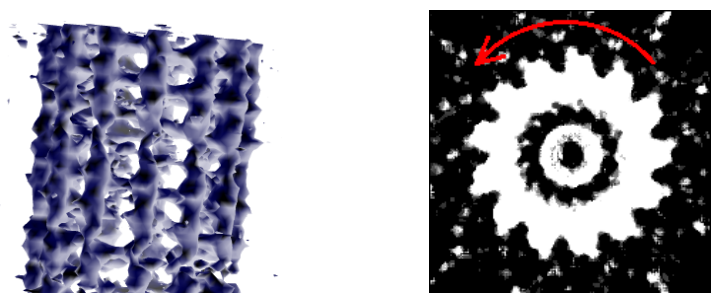

Fig. 5. Three dimensional reconstruction (left) of a microtubule visualized with CHIMERA (www.cgl.ucsf.edu/chimera/). Its axial projection view (right) shows a counterclockwise slew, which means that the microtubule it observed from its plus end [12].

\section{CONCLUSION}

To our knowledge, there is no method to automatically straighten non centro-symmetric helices (all "uneven" helices). In this paper, we propose a new algorithm to determine local centers of centro and non centro-symmetric microtubule images. This algorithm is planned to be freely available as a user friendly plug-in for the ImageJ software. Our method relies on the global cylindrical shape of the microtubules, and can easily be extended to other helical polymers. The only limitation is that no Bessel function significantly overlap the zero-oder Bessel function, at least close to the center of the fourier transform.

Experimental results showed that centers are estimated with an accuracy of lower than 0.5 pixel on synthetic data. The method was also applied on real images of centro and non centro-symmetric microtubules to automatically straighten them, and experts validated these results. Straightened microtubule images can be easily filtered, or used for three dimensional reconstruction.

\section{REFERENCES}

[1] E. H. Egelman, "An algorithm for straightening images of curved filamentous structures," Ultramicroscopy, vol. 19, no. 4, pp. 367-373, 1986.

[2] C.H. Owen, D.G. Morgan, and D.J. DeRosier, "Image analysis of helical objects: The brandeis helical package," J. Structural Biology, vol. 116, no. 1, pp. 167-175, January 1996.

[3] Z. Metlagel, Y.S. Kikkawa, and M. Kikkawa, "Ruby-helix: an implementation of helical image processing based on object-oriented scripting language," J. Structural Biology, vol. 157, no. 1, pp. 95-105, August 2007.

[4] E. Kocsis, B. L. Trus, C. J. Steer, M. E. Bisher, and A. C. Steven, "Image averaging of flexible fibrous macromolecules: the clathrin triskelion has an elastic proximal segment," J. Structural biology, vol. 107, no. 1, pp. 6-14, August 1991.

[5] M. F. Moody, "Image analysis of electron micrographs," in Biophysical Electron Microscopy: Basic Concepts and Modern Techniques, P. W. Hawkes and U Valdré, Eds., chapter 7, pp. 145-287. Academic Press, 1991.

[6] M. Stewart, "Computer image processing of electron micrographs of biological structures with helical symmetry," J. Electron Microscopy Technique, vol. 9, no. 4, pp. 325-58, August 1988 .

[7] D. J. DeRosier and A. Klug, "Reconstruction of three dimensional structures from electron micrographs," Nature, vol. 217, pp. 130-134, 1968.

[8] D. Toth, T. Aach, and V. Metzler, "Bayesian spatio-temporal motion detection under varying illumination," in European Signal Processing Conference (EUPSICO), Tampere , Finlande, 2000, pp. 2081-2084.

[9] T. Tasdizen, E. Jurrus, and R.T. Whitaker, "Non-uniform illumination correction in transmission electron microscopy images," in MICCAI Workshop on Microscopic Image Analysis with Applications in Biology (MIAAB08), New York, USA, 2008.

[10] D. Chrétien and R. H. Wade, "New data on the microtubule surface lattice," Biology of the Cell, vol. 72, pp. 161-174, 1991.

[11] Kuglin C.D. and D.C. Hines, "The phase correlation image alignment method," in IEEE International Conference on Cybernetics and Society, 1975, p. 163165.

[12] H. Sosa and D. Chrétien, "Relationship between moiré patterns, tubulin shape, and microtubule polarity," Cell Motility and the Cytoskeleton, vol. 40, no. 1, pp. 38-43, 1998. 